www.jmscr.igmpublication.org

Impact Factor 5.84

Index Copernicus Value: 71.58

ISSN (e)-2347-176x ISSN (p) 2455-0450

crossref DOI: _https://dx.doi.org/10.18535/jmscr/v5i10.92

Journal Of Medical Science And Clinical Research

IGM Publication

An Official Publication of IGM Publication

\title{
To assess the prevalence of dry eye disease in postmenopausal females in a tertiary care centre in Central India
}

\author{
Authors \\ Dr Neha Adlakha ${ }^{1}$, Dr Eva Rani Tirkey ${ }^{2}$, Dr Sujata Lakhtakia ${ }^{3}$ \\ ${ }^{1}$ Senior Resident, ${ }^{2}$ Associate Professor, ${ }^{3}$ Assistant Professor \\ ${ }^{1,2,3}$ Department of Ophthalmology, S.S. Medical College and Gandhi Memorial Hospital, Rewa (M.P.) India \\ Corresponding Author \\ Dr Neha Adlakha \\ Address-House No-1544, Sector-7 Extension, Gurgaon (Haryana)-122001 India \\ Mobile number-8223856309, Email: neha.adlakha777@gmail.com
}

\begin{abstract}
Dry eye is disorder due to deficiency of tear film and is common amongst postmenopausal women. Aim is to establish occurrence and prevalence of dry eye in postmenopausal women in tertiary care centre in Central India. A Cross-sectional study was carried out on 150 post-menopausal women. All patients were recorded in a predesigned proforma and Ocular Surface Disease Index scoring was done. Visual acuity was assessed on Snellen's chart and anterior segment evaluation was done using slit lamp biomicroscopy.Dry eye evaluation was done with Schirmer Test 1 and Tear film Break-Up time test. Corneal and conjunctival staining using flouroscein and Rose Bengal was performed. Assessment of meibomian gland function and grading of dry eye was done according to DEWS classification. Data was analyzed using Statistical Package for Social Science (SPSS) standard version 13.0. T test. Chi square and Pearson correlation test was used for data analysis. Fifty two out of 150 post-menopausal women (34.6\%) were found to have dry eyes. Prevalence of dry eye was maximum in age group $>74$ years (38.46\%). Meibomian gland scores were normal in $28.84 \%$ of patients and abnormal in $71.15 \%$ patients. Most patients in present study had evaporative dry eye(46.15\%),followed by mixed type(40.38\%) and aqueous deficient(13.46\%).Most of the patients in our study had mild dry eye(44.23\%),followed by severe(32.69\%) and $23.07 \%$ accounting for moderate dry eye. Present study reveal that dry eye symptoms are common problems in postmenopausal women. Hence, examination for dry eye should be integral part of postmenopausal women especially those having dry eye symptoms. It is important for primary care physicians to understand DED due to its high prevalence and potentially preventable and treatable nature of the condition.

Keywords: Dry eye, meibomian, tear film, postmenopausal, prevalence, Schirmer's test.
\end{abstract}

\section{Introduction}

Dry eye disease is the most frequently encountered disorder in Ophthalmology. Dry eye per se is not a disease entity, but a symptom complex occurring as a sequelae to deficiency or abnormalities of tear film ${ }^{[1]}$. It is defined as a disorder of the tear film due to tear deficiency or excessive tear evaporation, which causes damage to the interpalpebral ocular surface and is associated with symptoms of ocular discomfort ${ }^{[2]}$. Dry eye disease (DED) is defined by the International Dry Eye Workshop as, “A 
multifactorial disease of the tears and ocular surface that results in symptoms of ocular discomfort, visual disturbance and tear film instability with potential damage to ocular surface $^{[3]}$. It is accompanied by an increased osmolarity of tear film and inflammation of ocular surface. It can cause debilitating symptoms including burning, foreign body sensation, decreased vision and affect activities of daily living. Different studies have found a relatively wide range of prevalence estimates, ranging from $7 \%$ to $33 \%{ }^{[4]}$.The Ocular Surface Disease Index (OSDI) is a questionnaire used to evaluate symptoms of dry eye. There have been few studies which have supported the role of OSDI for the diagnosis of dry eye syndrome ${ }^{[6]}$. Postmenopausal women have higher incidence of DED. Large scale epidemiological studies done in United States have shown that rate of DED in women over 50 years old is nearly double that in men over 50 at $7 \%$ and $4 \%$, respectively ${ }^{[8]}$. Numerous studies have demonstrated that there is a hormonal etiology behind this group's susceptibility to DED. Androgens have been proven to have an effect on tear production and function ${ }^{[9]}$. The hormonal changes may lead to decreased tear production which is the root of dry eye syndrome. About $60 \%$ of menopausal women are affected by dry eyes. The symptoms of dry eye, namely dryness, itchiness, photophobia, blurring of vision, foreign body sensation and tearing may cause considerable discomfort to patients and subsequently interfere with their quality of life. Untreated dry eye syndrome increase the susceptibility of the eyes towards infection as well as causing serious visual impairment ${ }^{[11]}$. Hormone Replacement Therapy has been well accepted to improve the quality of life among the postmenopausal women ${ }^{[12]}$. After menopause, the level of estrogen in the blood decreases. Kramer had shown that the eye is a locus of action of female sexual hormones. Sator proposed the reduction of naturally occurring estrogen as a possible reason for the occurrence of dry eye in menopausal women. Sex steroid receptors are present on the meibomian glands, which are the sebaceous glands on the eyelids responsible for producing the oil component of tears that prevents evaporation ${ }^{[13]}$. Androgen binding results in synthesis and secretion of lipids from these glands, while estrogens actually cause a decrease in lipid production ${ }^{[14]}$. For this reason, increased levels of estradiol are believed to be a risk factor for dry eye. However, the exact relationship between serum sex hormone levels and clinical symptoms of dry eye remains unclear and controversial. Various studies have shown that postmenopausal dry eye is clearly not limited to aqueous deficiency and inflammatory changes of the ocular surface and lacrimal gland. Oestrogen deficiency may lead to sebaceous gland alteration, so further destabilization of the tear film occurs due to meibomian gland dysfunction. Studies have shown that oestrogen receptor mRNA are present in lacrimal gland, meibomian gland, lids, palpebral and bulbar conjunctiva, cornea and other anterior ocular surface. Their presence in the ocular structures and the observation that dry eye syndrome is prevalent in the menopausal and perimenopausal population implies that tear film function is under a complex hormonal influence. Novel androgen formulations that can be given topically to the eyelids or as eye drops, have shown promising results with decreased systemic side effects ${ }^{[15,16]}$ Given high prevalence of DED, it is important for gynecologists and for primary care physicians to understand DED to recognize symptoms and start initial lubricating therapy and identify patients who would benefit from Ophthalmology referral for further intervention. Thus this study was done to establish the occurrence and prevalence of dry eye and meibomian gland dysfunction in postmenopausal women in a tertiary care centre in Central India and also to establish the effect of age on dry eye.

\section{Objectives}

1. To assess the prevalence of dry eye in post-menopausal females. 
2. To assess the prevalence of dry eyes with relation to the age of post-menopausal females.

3. To assess the meibomian gland dysfunction in post-menopausal females

4. To assess the prevalence of various types of dry eye in post-menopausal females.

\section{Materials and Methods}

This observational cross-sectional study was conducted on 150 postmenopausal females attending indoor and outpatient of Department of Ophthalmology. This research work was executed after approval from the Institutional Ethical Committee. All the patients were recorded in a predesigned proforma, including the detailed history and relevant examination and were informed about the procedure and a written consent was taken. The inclusion criteria were postmenopausal women who had completely achieved natural menopause of at least one year. Patients with systemic disorders known to cause dry eyes such as Rheumatoid Arthritis, Diabetes Mellitus, Thyroid Disease, AIDS, Graft Versus Host Disease, Parkinson's Disease, patients who had underwent bone marrow transplant, patients on medications known to cause dry eyes like anticholinergic medications, Non-steroidal Anti Inflammatory Drugs, Beta-blocker and radiation therapy, patients with known ocular surface diseases like Herpes Simplex Keratitis and Herpes Zoster Ophthalmicus, topical eye drop users and patients who had recent ocular surgery within 3 months and contact lens wearer were excluded. Ocular surface Disease Index (OSDI) questionnaire was completed by all patients and grading done. Visual acuity was assessed on Snellen's chart and anterior segment evaluation was done with slit lamp biomicroscope. Corneal and conjunctival staining using flouroscein and Rose Bengal was performed. Dry eye evaluation was done with Schirmer and Tear film Break-Up time test. Schirmer test which is a useful assessment of aqueous tear production and is performed by placing a special filter paper
(Whatmann Filter paper No.:41) in the lower fornix was done. Aqueous Tear film Break-Up Time was done by an impregnated fluorescein strip moistened with non-preserved saline instilled into the lower fornix. The TBUT of less than 10 seconds was noted. Assessment of meibomian gland function was done by mebum quality, expressibility and MGYLS (Number of Meibomian glands yielding liquid secretions). Meibomian gland status grading was done according to The International Workshop on Meibomian Gland Dysfunction 2011. Patients were graded according to 4 point severity scale for dry eye recommended by DEWS guidelines modified by Asbell and Lemp 2013. Data was analyzed using the Statistical Package for Social Science (SPSS) standard version 13.0. T test. Chi square and Pearson correlation test was used for data analysis. A value of $\mathrm{p}<0.05$ was considered statistically significant.

\section{Observations and Results}

This prospective observational study was undertaken on 150 postmenaupausal females attending indoor and outpatient of Department of Ophthalmology. Out of 150,52 patients $(34.66 \%)$ had dry eye(Table 1)

Percentage prevalence of dry eye was maximum in the age group $>74$ years $(38.46 \%)$ and minimum in the age group 45-49 years. (Table 2) Dry eye severity grading was done according to all diagnostic tests. Most of the patients in our study had mild dry eye(44.23\%), followed by severe $(32.69 \%)$ and then moderate $(23.07 \%)$ dry eye. (Table-3)

Meibomian gland scores were normal in 44 eyes $(29.33 \%)$ and abnormal in 106 patients $(70.66 \%)$ patients (Table 4)

Most patients in present study had evaporative dry eye $(46.15 \%)$, followed by mixed $(40.38 \%)$ and aqueous deficient $(13.46 \%)$ (Table 5)

Table 1 shows prevalence of dry eye among postmenopausal females

\begin{tabular}{|l|c|c|}
\hline $\begin{array}{l}\text { Total Number Of } \\
\text { Patients }\end{array}$ & $\begin{array}{c}\text { No. Of Patients Of } \\
\text { Dry Eye }\end{array}$ & Percentage \\
\hline $\mathbf{1 5 0}$ & 52 & $34.66 \%$ \\
\hline
\end{tabular}


Table 2 shows percentage prevalence of dry eye in each age group

\begin{tabular}{|l|c|c|}
\hline $\begin{array}{l}\text { Age Group } \\
\text { (In Years) }\end{array}$ & $\begin{array}{c}\text { No. Of } \\
\text { Patients }\end{array}$ & $\begin{array}{c}\text { Percentage Prevalence Of } \\
\text { Dry Eye (\%) }\end{array}$ \\
\hline $\mathbf{4 5 - 4 9}$ & 15 & 26.66 \\
\hline $\mathbf{5 0 - 5 4}$ & 19 & 31.57 \\
\hline $\mathbf{5 5 - 5 9}$ & 23 & 34.78 \\
\hline $\mathbf{6 0 - 6 4}$ & 31 & 35.48 \\
\hline $\mathbf{6 5 - 6 9}$ & 33 & 36.36 \\
\hline $\mathbf{7 0 - 7 4}$ & 16 & 37.50 \\
\hline$>\mathbf{7 4}$ & 13 & 38.46 \\
\hline
\end{tabular}

Table 3 shows grading of severity of dry eye

\begin{tabular}{|l|c|c|}
\hline Grading Of Dry Eye & No. Of Patients & Percentage \\
\hline Mild & 23 & $44.23 \%$ \\
\hline Moderate & 12 & $23.07 \%$ \\
\hline Severe & 17 & $32.69 \%$ \\
\hline
\end{tabular}

Table 4 shows meibomian gland scores in dry eye

\begin{tabular}{|l|c|c|}
\hline $\begin{array}{l}\text { Meibomian Gland } \\
\text { Score }\end{array}$ & $\begin{array}{c}\text { Number of } \\
\text { Patients }\end{array}$ & Percentage \\
\hline Normal(1) & 15 & $28.84 \%$ \\
\hline Abnormal(>1) & 37 & $71.15 \%$ \\
\hline
\end{tabular}

Table 5 shows percentage prevalence of various types of dry eye

\begin{tabular}{|l|c|c|}
\hline Type Of Dry Eye & Number Of Patients & Percentage \\
\hline Aqueous Deficient & 7 & $13.46 \%$ \\
\hline Evaporative & 24 & $46.15 \%$ \\
\hline Mixed & 21 & $40.38 \%$ \\
\hline
\end{tabular}

\section{Discussion}

Kerato conjunctivitis sicca or dry eye refers to a variety of disorders, characterised by ocular surface disease that results from any condition that decreases tear secretion and/or increases tear film evaporation sufficient to result in loss of water from tear film. Dry eye is a potentially serious and chronic condition that may limit a person's activity and in extreme cases, causes blindness. After menopause, hormonal imbalance leads to development of dry eye. Hence, examination of dry eye and meibomian gland dysfunction should be an integral part of postmenopausal women especially those having dry eye symptoms.

In present study, prevalence of dry eye in postmenopausal women was $34.66 \%$.Our finding was closely related to study done by B. Shaharuddin et al $(2008)^{[17]}$ in which prevalence was $29 \%$. In our study, prevalence of dry eye was maximum in age group greater than 74 years $(38.46 \%)$ and we also found that prevalence increases progressively with age. These results were consistent with study done by Anshu Sahai et al (2005) ${ }^{[18]}$ Ram S Mirley (2000) suggested that both tear flow rate and tear stability reduces with age and by the age of 40 years, tear production is reduced to $50 \%$ of that at the age of 10 years. Reduction of tear volume and flow and increase in evaporation have been noted in elderly as seen in study of Mathers et al(1996) ${ }^{[20]}$.In another study of dry eye syndrome among US women showed that the prevalence of DES increased with age, from $5.7 \%$ among women < 50 years old to $9.8 \%$ among women aged $\geq 75$ years old. The age-adjusted prevalence of DES was $7.8 \%$, or 3.23 million women aged $\geq 50$ in the US. The prevalence of DED is known to increase with age There are various hypotheses to support this, one important hypothesis being age-related decrease in meibomian gland secretion possibly due to atrophy of acinar cells, similar to agerelated decline in the functioning of other sebaceous glands. Also aging is associated with alterations in the quality of meibomian gland secretions.

In present study, we found abnormal meibomian gland score $(>1)$ in 30 patients $(28.84 \%$ ),probably due to detrimental effects of estrogen or androgen or both as sex hormone influences both lacrimal and meibomian glands. According to Uchino $\mathrm{M}$ et $\operatorname{al}(2006)^{[22]}$, meibomian gland dysfunction is an important factor in relation to dry eye disease with meibomian gland dropout showing positive correlation with tear film instability. In present study, we found evaporative dry eye accounts for maximum contribution (46.15\%), followed by mixed $(40.38 \%)$ and minimum by aqueous deficient (13.46\%). Similar finding was observed by Kelly Nichols (2013) ${ }^{[23]}$. Most common type of dry eye in post menopausal women is of evaporative type possibly due to meibomian gland dysfunction and its decreased secretion due to atrophy of acinar cells (declined functioning of 
sebaceous glands) due to hormonal imbalance particularly androgen deficiency. These results were consistent with study done by Debra A. Schaumberg et al (2001) ${ }^{[24]}$. The data from a study done by Caterina Gagliano et al ${ }^{[25]}$ suggest that deficiency in sexual hormones may cause not only a reduction in tear production leading to aqueousdeficient dry eye, but also a dysfunction in meibomian gland function determining an evaporative dry eye. In our study, most of the patients had mild dry eye $(44.23 \%)$ followed by severe $(32.69 \%)$ and $25 \%$ contributed to moderate type, as per all diagnostic tests (Schirmer's test, TFBUT, vital corneal and conjunctival dye stains)

\section{Conclusion}

Postmenopausal dry eye has now evolved as a significant problem in recent years as its prevalence is very high and it is difficult to treat condition in late stages. Reason being hormonal changes, under diagnosis of mild to moderate cases and poor compliance of patients in this age group. Alteration of sex hormones plays an important role in the pathophysiology of DED in perimenopausal and menopausal age group. Often, simple measures such as lubrication may provide relief. In more severe cases, anti-inflammatory, immunomodulatory, and rarely surgical interventions are required. Novel hormonal replacement treatments, both systemic and topical, are also evolving. Hence, patients presenting to Gynaecology OPD with post-menopausal complaints should also be screened for ocular complaints and referred to Ophthalmology OPD to assist early detection and avoid debility and possible blindness due to dry eye related complications

\section{Acknowledgements: Nil}

Conflicting Interest (If present, give more details): NIL

Source(s) of support: NIL

\section{References}

1. Sihota R, Tandon R. Systemic Ophthalmology. In: Sihota R, Tandon R, editors. Parson's Diseases of the Eye, 21st ed. New Delhi: Elsevier; 11.p.463-464

2. Lemp MA. Report of the National Eye Institute/Industry Workshop on clinical trials in dry eyes. CLAO J 1995;21:221-32

3. The definition and classification of dry eye disease: Report of the Definition and Classification Subcommittee of the International Dry Eye WorkShop (2007).Ocul Surf 2007;5:75-92

4. Lin PY, Tsai SY, Cheng CY, Liu JH, Chou P, Hsu WM. Prevalence of dry eye among an elderly Chinese population in Taiwan: The Shihpai Eye Study. Ophthalmology $2003 ; 110: 1096-101$

5. Ozcura F, Aydin S, Helvaci MR. Ocular surface disease index for the diagnosis of dry eye syndrome. Ocul Immunol Inflamm. 2007 Sep-Oct; 15 (5): 389-93.

6. Schaumberg DA, Sullivan DA, Buring JE, Dana MR. Prevalence of dry eye syndrome among US women. Am J Ophthalmol 2003; 136:318-26

7. Ablamowicz AF, Nichols JJ, Nichols KK. Association between serum levels of testosterone and estradiol with meibomian gland assessments in postmenopausal women. Invest Ophthalmol Vis Sci 2016;57:295-300

8. Lemp MA. Report of the National Eye Institute/Industry workshop on Clinical Trials in Dry Eyes. CLAO J 1995;21(4):221-32

9. Genazzani AR, Nicolucci A, Campagnoli C, Crosignani P, Nappi C, Serra GB, et al. Assessment of the QoL in Italian menopausal women: comparison between HRT users and non-users. Maturitas 2002;42(4):267-80.

10. Dawson T. Testosterone eye drops: A novel treatment for dry eye disease. Ophthalmology Times; 2015.

11. Suzuki T, Schirra F, Richards SM, Jensen RV, Sullivan DA.Estrogen and progesterone control of gene expression in 
the mouse meibomian gland. Invest Ophthalmol Vis Sci2008;49:1797-808

12. Conner CG. Symptomatic relief of dry eye assessed with the OSDI in patients using $5 \%$ testosterone cream. Invest Ophthalmol Vis Sci 2005;46:2032.

13. Dawson T. Testosterone eye drops: A novel treatment for dry eye disease. Ophthalmology Times; 2015

14. Shaharuddin B, Ismail-Mokhtar S, Hussein E. Dry eye in post-menopausal Asian women on hormone replacement therapy. Int J Ophthalmol 2008; 1: 158-160

15. Anshu Sahai, MS; Pankaj Malik, DNB. Dry eye: Prevalence and attributable risk factors in a hospital based population. Indian Journal of Ophthalmology 2005;53:87-91

16. Mathers WD, Daley TE. Tear flow and evaporation in patients with and without dry eye. Ophthalmology. 1996;103:664669

17. Uchino M Dogru M Yagi Y The features of dry eye disease in a Japanese elderly population. Optom Vis Sci . 2006; 83: 797802

18. Narayanan S, Redfern RL, Miller WL, Nichols KK, McDermott AM. Dry eye disease and microbial keratitis: is there a connection? Ocul Surf. 2013;11(2):75-92

19. Sullivan, D. A., Sullivan, B. D., Evans, J. E., Schirra, F., Yamagami, H., Liu, M., Richards, S. M., Suzuki, T., Schaumberg, D. A., Sullivan, R. M. And Dana, M. R. (2002), Androgen Deficiency, Meibomian Gland Dysfunction, and Evaporative Dry Eye. Annals of the New York Academy of Sciences, 966: 211-222

20. Caterina Gagliano, Salvatore Caruso, Giuseppe Napolitano, Giulia Malaguarnera. Low levels of 17- $\beta$-oestradiol, oestrone and testosterone correlate with severe evaporative dysfunctional tear syndrome in postmenopausal women: a case-control study. Br J Ophthalmol 2014; 98: 371-376. 